\title{
Homotopy Analysis Method for a Class of Holling Model with the Func- tional Reaction
}

\author{
Xiurong Chen ${ }^{*}$ and Jiaju Yu
}

School of Science and Information, Qingdao Agricultural University, Qingdao, China

\begin{abstract}
In this paper, the homotopy analysis method is proposed to solve a class of holling model with the functional reaction. The model is characterized with the nonlinear equations in the denominator and it is difficult to obtain the closed approximation solutions. The series solutions of the model are obtained and the results show that the presented method is efficiency and simplicity. The convergence of this algorithm is also proved.
\end{abstract}

Keywords: Holling Model, Homotopy Analysis Method, Approximation Solutions.

\section{INTRODUCTION}

The prey-predator system is a very important model that has been studied by many ecologists and mathematicians. Recently, the holling model with functional reaction becomes a very attractive topic. Many works [1-5] focused on discussing the stability and the limit cycles, and so on. The direct and simple numerical simulations are lack. So finding explicit analytic solutions of system is extremely important in biology.

It is difficult to get ecological model's solution because of nonlinearity in the denominator. In recent years, many powerful methods have been developed to construct explicit analytic solution of nonliear system. In 1992, Liao [6] employed a general analytic method for nonlinear problems, namely homotopy analysis method (HAM). This technique has successfully been applied to solve many nonlinear problems, such as the $\mathrm{KdV}$-type equations [7, 8], nonlinear heat transfer [9], nonlinear water equations [10], differential difference equations [11], and so on. The validity of HAM is independent of whether or not there exists small parameter in the considered equation. Besides, different from all previous numerical and analytical methods, it contains a certain auxiliary parameter $h$, which provides us with a simple way to adjust and control the convergence of solution series.

In this work, the HAM is used to solve the predator-prey models. The rest of this paper is organized as follows. In section 2, a brief introduction about the method is provided, and we provide the convergent theorem for nonlinear equation. In section 3, Holling III's model, is documented, the obtained results suggest that newly improvement technique is promising tool and powerful improvement for nonlinear equations. The last section includes our conclusions.

*Address correspondence to this author at the School of Science and Information, Qingdao Agricultural University, Qingdao, China;

Tel: +186 13475809936; E-mail: xrchen_100@163.com

\section{BASIC IDEAS OF HAM}

In this section, we give some basic concepts of the homotopy analysis method. To do this, consider the following nonlinear equation

$$
N[u(t)]=0
$$

where $N$ is a nonlinear operator, $t$ denote independent variables, and $u(t)$ is an unknown function, respectively. For simplicity, we ignore all boundary or initial conditions, which can be treated in a similar way. With the generalized traditional homotopy method, Liao [6] constructs the socalled zero-order deformation equations

$(1-p) L\left[\varphi(t ; p)-u_{0}(t)\right]=p \hbar H(t) N[\varphi(t ; p)]$

where $p \in[0,1]$ is an embedding parameter, $\hbar \neq 0$ is an auxiliary parameter, $H(t) \neq 0$ an auxiliary function, $L$ is an auxiliary linear operator. $u_{0}(t)$ is an initial guess of $u(t)$, $\varphi(t ; p)$ is an unknown function, respectively. It is important that one has great freedom to choose auxiliary things in HAM. Obviously, when $p=0$ and $p=1$,

$\varphi(t, 0)=u_{0}(t) ; \quad \varphi(t, 1)=u(t)$

Therefore, as $p$ increases from 0 to $1, \varphi(t ; p)$ vary or deform from the initial $u_{0}(t)$ to the exact solution $u(t)$ governed by Eq. (1). This is the basic idea of the homotopy and this kind of variation is called deformations in topology. Thus, by Taylor's theorem and Eq. (3), we can express

$\varphi(t ; p)=u_{0}(t)+\sum_{k=1}^{\infty} u_{k}(t) p^{k}$

where 
$u_{k}(t)=\left.\frac{1}{k !} \frac{\partial^{k} \varphi(t ; p)}{\partial p^{k}}\right|_{p=0}$

If the auxiliary linear operator, the initial guess and the auxiliary parameter $h$ are so properly chosen, Eq. (4) is convergent at $p=1$, then one has

$u(t)=\varphi(t, 1)=u_{0}(t)+\sum_{k=1}^{\infty} u_{k}(t)$

which must be one of the solutions of the original nonlinear Eq. (1), as proved by Liao [6]. Now, define the vectors

$\vec{u}_{n}=\left\{u_{0}(t), u_{1}(t), \cdots, u_{n}(t)\right\}$

Differentiating the zero-order deformation Eq. (2) $\mathrm{m}$ times with respect to $t$ and then dividing them by $m$ ! and finally setting $p=0$, we have the mth-order deformation equations

$L\left[u_{m}(t)-\chi_{m} u_{m-1}(t)\right]=h H(t) R\left(\vec{u}_{m-1}\right)$

where

$$
R\left(\vec{u}_{m-1}\right)=\left.\frac{1}{(m-1) !} \frac{\partial^{m-1} N[\varphi(x, t ; p)]}{\partial p^{m-1}}\right|_{p=0}
$$

and

$\chi_{m}= \begin{cases}0 & m \leq 1 \\ 1 & m>1\end{cases}$

The solution of the mth-order deformation Eq. (8) is readily found to be

$u_{m}(t)=\chi_{m} u_{m-1}(t)+h H(t) L^{-1}\left[R\left(\vec{u}_{m-1}\right)\right]$

It should be emphasized that $u_{i}(t)$ is governed by Eq. (7) with the initial conditions that come from the original problems, which can be easily solved by symbolic computation software such as Maple and Mathematics.

\section{CONVERGENCE THEOREM}

Theorem If the series (6), i.e. $\sum_{k=0}^{\infty} u_{k}(t)$ converge to function $u(t)$, then $u(t)$ must be the exact solution of Eq. (1).

Proof Since the series $\sum_{k=0}^{\infty} u_{k}(t)$ is convergent, we must have

$\lim _{n \rightarrow \infty} u_{n}(t)=0$

Using the definition (10), we have

$\sum_{m=1}^{n}\left[u_{m}(t)-\chi_{m} u_{m-1}(t)\right]=u_{1}(t)+\left[u_{2}(t)-u_{1}(t)\right]$

$+\cdots+\left[u_{n}(t)-u_{n-1}(t)\right]=u_{n}(t)$
Therefore from (12), we lead to

$\sum_{m=1}^{\infty}\left[u_{m}(t)-\chi_{m} u_{m-1}(t)\right]=\lim _{n \rightarrow \infty} u_{n}(t)=0$

Now, from the above expression and (11) we have

$h H(t) \sum_{m=1}^{\infty}\left[R_{1 m}\left(u_{m-1}\right)\right]=$

$\sum_{m=1}^{\infty}\left[u_{m}(t)-\chi_{m} u_{m-1}(t)\right]=0$

Since $h \neq 0$ and $H(t) \neq 0$ then the above equation gives

$\sum_{m=1}^{\infty}\left[R_{1 m}\right]=0$

Now, from (1) and definitions (5) and (9), it holds that

$$
\begin{aligned}
& \sum_{m=1}^{\infty}\left[R_{m}\right]=\left.\sum_{m=1}^{\infty} \frac{1}{(m-1) !} \frac{\partial^{m-1} N[\varphi(x, t ; p)]}{\partial p^{m-1}}\right|_{p=0} \\
& =N\left[\left.\sum_{m=1}^{\infty} \frac{1}{(m-1) !} \frac{\partial^{m-1} \varphi(x, t ; p)}{\partial p^{m-1}}\right|_{p=0}\right] \\
& =N[u(t)]=0
\end{aligned}
$$

In the above equations we used from this fact that $L$ is a linear operator and the series $\sum_{k=0}^{\infty} u_{k}(t)$ converges to $u(t)$, respectively. Hence, $u(t)$ are the exact solutions of Eq. (1) and the proof is completed.

\section{APPLICATION TO THE MODEL}

Now we consider the Holling III' model [12]:

$$
\left\{\begin{array}{l}
x^{\prime}=r x-\frac{\alpha x^{2} y}{1+\omega x^{2}} \\
y^{\prime}=y\left(-d+e \frac{\alpha x^{2}}{1+\omega x^{2}}-b y\right)
\end{array}\right.
$$

Here $x(t), y(t)$ represent the population density of two species respectively at time $t$, a susceptible pest and infected pest which live on the crop. All parameters are positive constants which meaning omitted here (see [12]). Type on both sides of the Eq. (17) with $1+\omega x^{2}$, note $\omega=a_{1}, r \omega=a_{2}, \alpha=a_{3}, e \alpha-d \omega=b_{1}, b \omega=b_{2}$, this leads to the dimensionless equation

$\left\{\begin{array}{l}x^{\prime}-r x+a_{1} x^{2} x^{\prime}-a_{2} x^{3}+a_{3} x^{2} y=0 \\ y^{\prime}+d y+b y^{2}+a_{1} x^{2} y^{\prime}-b_{1} x^{2} y+b_{2} x^{2} y^{2}=0\end{array}\right.$

with the initial conditions $x(0)=A, y(0)=B$. Due to the governing Eq. (18), the auxiliary linear operators are choosed:

$$
\begin{aligned}
& L_{1}[X(t ; p)]=\frac{\partial X(t ; p)}{\partial t}+X(t ; p) \\
& L_{2}[Y(t ; p)]=\frac{\partial Y(t ; p)}{\partial t}+Y(t ; p)
\end{aligned}
$$


which satisfy $L_{1}\left[C_{1} e^{-t}\right]=0, L_{2}\left[C_{2} e^{-t}\right]=0$, where $C_{1}, C_{2}$ are integral constants, and the $X(t ; p), Y(t ; p)$ are unknown real functions. Furthermore, due to (18), the non-linear operators are defined

$$
\begin{aligned}
& N_{1}[X(t ; p), Y(t ; p)]=\frac{d X}{d t}-r X+a_{1} X^{2} X^{\prime} \\
& -a_{2} X^{3}+a_{3} X^{2} Y . N_{2}[X(t ; p), Y(t ; p)]=\frac{d Y}{d t}+d Y+b Y^{2}+ \\
& a_{1} X^{2} Y^{\prime}-b_{1} X^{2} Y+b_{2} X^{2} Y^{2}
\end{aligned}
$$

Then, introducing a non-zero auxiliary $h$, the zero-order deformation equations

$(1-p) L_{1}\left[X(t ; p)-x_{0}(t)\right]=$

$h_{1} H_{1}(t) p N_{1}[X(t ; p), Y(t ; p)]$

$(1-p) L_{2}\left[Y(t ; p)-y_{0}(t)\right]=$

$h_{2} H_{2}(t) p N_{2}[X(t ; p), Y(t ; p)]$

Obviously, when $p=0$ and $p=1$,

$$
\begin{aligned}
& X(t ; 0)=x_{0}(t), Y(t ; 0)=y_{0}(t) \\
& X(t ; 1)=x(t), Y(t ; 1)=y(t)
\end{aligned}
$$

Therefore, as the embedding parameter $p$ increases from 0 to $1, X(t ; p), Y(t ; p)$ vary from the initials $x_{0}(t), y_{0}(t)$ to the exact solution $x(t), y(t)$ governed by (13). This is the basic idea of the homotopy and this kind of variation is called deformations in topology. Expanding $X(t ; p)$ and $Y(t ; p)$ in Taylor series with respect to $p$ admits

$$
\begin{aligned}
& X(t, p)=x_{0}(t)+\sum_{k=1}^{\infty} x_{k}(t) p^{k} \\
& Y(t, p)=y_{0}(t)+\sum_{k=1}^{\infty} y_{k}(t) p^{k}
\end{aligned}
$$

where

$$
\left\{\begin{array}{l}
x_{k}(t)=\left.\frac{1}{k !} \frac{\partial^{k} X(t ; p)}{\partial p^{k}}\right|_{p=0} \\
y_{k}(t)=\left.\frac{1}{k !} \frac{\partial^{k} Y(t ; p)}{\partial p^{k}}\right|_{p=0}
\end{array}\right.
$$

If the auxiliary linear parameter, the initial conditions, and the auxiliary parameters $h_{1}=h_{2}=h, H_{1}(t)=H_{2}(t)$ $=1$ are chosen, the above series converge at $p=1$, and one has

$$
x(t)=x_{0}(t)+\sum_{k=1}^{\infty} x_{k}(t), y(t)=y_{0}(t)+\sum_{k=1}^{\infty} y_{k}(t)
$$

Differentiating the zero-order deformation equations (19) $m$ times with respect to $p$ and then dividing them by $m$ ! and finally setting $p=0$, the mth-order deformation equations read

$$
\begin{aligned}
& L_{1}\left[x_{m}(t)-\chi_{m} x_{m-1}(t)\right]=h R_{1 m}\left[x_{m-1}, y_{m-1}\right] \\
& L_{2}\left[y_{m}(t)-\chi_{m} y_{m-1}(t)\right]=h R_{2 m}\left[x_{m-1}, y_{m-1}\right]
\end{aligned}
$$

Where

$$
\begin{aligned}
& R_{1 m}(t)=x_{m-1}^{\prime}-r x_{m-1}-\sum_{i=0}^{m-1}\left[a_{1} \sum_{j=0}^{i} \frac{d x_{i}}{d t} x_{i-j}\right. \\
& \left.-a_{2} \sum_{j=0}^{i} x_{j} x_{i-j}+a_{3} \sum_{j=0}^{i} x_{j} y_{i-j}\right] x_{m-1-i} \\
& R_{2 m}(t)=y_{m-1}^{\prime}+d y_{m-1}+b \sum_{i=0}^{m-1} y_{i} y_{m-1-i}+\sum_{i=0}^{m-1}\left[\sum_{j=0}^{i} \frac{d y_{j}}{d t} x_{i-j}\right. \\
& \left.+b_{1} \sum_{j=0}^{i} x_{i} y_{j-i}+b_{2} \sum_{j+n+l=0}^{i} x_{j} x_{n} y_{l}\right] x_{m-1-i}
\end{aligned}
$$

Now, the solutions of the mth-order deformation Eqs. (23) for $m \geq 1$ become

$$
\begin{aligned}
& x_{m}=\chi_{m} x_{m-1}+h \int_{0}^{t} \exp [r(t-s)] R_{1 m}(s) d s+c_{1} e^{-t} \\
& y_{m}=\chi_{m} y_{m-1}+h \int_{0}^{t} \exp (d(s-t)) R_{2 m}(s) d s+c_{2} e^{-t}
\end{aligned}
$$

Where the constants $c_{1}$ and $c_{2}$ are determined by the initial conditions $x_{m}(0)=0, y_{m}(0)=0$. Mathematica software is used to solve the linear Eqs. (25) under the initial conditions up to first few order of approximations. We have

$$
\begin{aligned}
& x(t)=x_{0}(t)+x_{1}(t)+\cdots=A e^{-t}-A_{11} e^{-t} h \\
& +A_{12} e^{-t} h t+A_{11} e^{-3 t} h+\cdots \\
& y(t)=y_{0}(t)+y_{1}(t)+\cdots=B e^{-t}+B_{11} e^{-3 t} h \\
& -B_{12} e^{-t} h-A_{12} e^{-t} h t+\cdots
\end{aligned}
$$

where

$$
\begin{aligned}
& A_{11}=\left[A\left(a_{1}+a_{2}\right)-a_{3} B\right] / 2, A_{12}=A(1+r) ; \\
& B_{11}=A_{11} A^{2}, B_{12}=A^{2}\left[a_{3}-A\left(a_{1}+a_{2}\right)\right] / 2 .
\end{aligned}
$$

The solutions obtained by (25) contain the parameter $h$. We plot the so-called $h$-curves to ensure solution series converge, as suggested by Liao [6]. The valid region of $h$ is a horizontal line segment. Thus the valid region of $h$ in this case is $-1.2<h<-0.8$, as shown in Fig. (1) when $r=0.1, b_{1}=0.02, A=0.3, B=0.5, a_{1}=0.3, t=0.01$ $b_{2}=0.003, b=0.011, a_{2}=0.002, a_{3}=0.01$, 


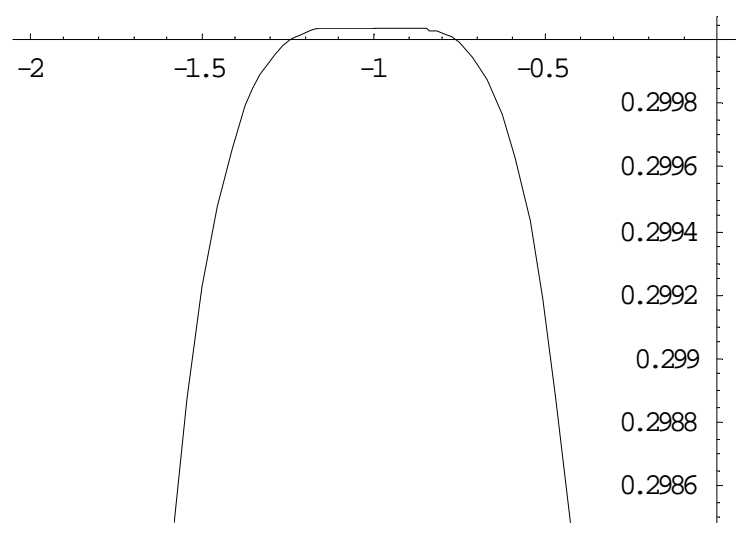

(a) $h$-curves of the 4-th order approximation for $x$

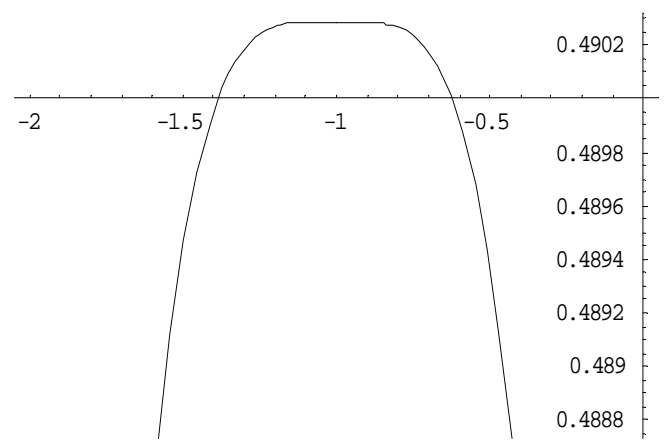

(b) $h$-curves of the 4-th order approximation for $y$

Fig. (1). the $h$-curves of the 4-th order approximation for $x(t)$ and $y(t)$.

$d=0.015$. So and we can discuss the qualitative of Eq. (18).

\section{CONCLUSIONS}

The ordinary equations which arise from ecologists problems are usually nonlinear and such equations with Functional Reaction are difficult to estimate numerically or analytically. In this paper the HAM was applied to solve the prey-predator system and the approximate analytic solutions are obtained. We can study the model's property by the approximate analytic solutions. This approach was very efficient and powerful technique in finding the solutions of the proposed equations.

\section{CONFLICT OF INTEREST}

The authors confirm that this article content has no conflicts of interest.

\section{ACKNOWLEDGEMENTS}

Declared none.

\section{REFERENCES}

[1] L. Jian-min, "Qualitative analysis of the secons kind functional reaction model of two species being harvested simultaneously", Journal of Henan University, vol. 34, no.3, pp. 12-15, 2004.

[2] C. Liu-juan and S. Jian-hua, "A qualitative analysis of system with the holling II functional response of predator to prey", Journal of Biomathematics, vol.18, no.1, pp. 33-36, 2003.

[3] Q. Weigen and L. Chuanrong, "The qualitative analysis of a preypredator system of holling's II functional response with havesting term", Mathematica Acta Fcientia, vol.19, no.1, pp.10-16, 1999.

[4] W. Yuming, "Qaulitative analysis of a kind of predator- prey system exploited", Guangxi Sciences, vol.10, no.1, pp.11-14, 2003.

[5] Y. Xiangping and Z. Xianghua, "The qualitative analysis of a class of two species predator- prey model with functional response", Journal of Biomathematics, vol.19, no.3, pp. 323-327, 2004.

[6] S.J Liao, "Homotopy analysis method and its application", $\mathrm{PhD}$ dissertation, Shanghai Jiao Tong University, 1992.

[7] S. Abbasbandy, "Solition solutions for the 5th-order KdVequation with the homotopy analysis method", Nnonlinear Dyn.51, pp. 8387, 2008.

[8] S. Abbasbandy, "The application of the homotopy analysis method to solve a generalized Hirota-Satsuma coupled KdV equation", Phys. Lett. A, vol. 361, pp. 478-483, 2007.

[9] S. Abbasbandy, "The application of the homotopy analysis method to solve a nonlinear equations arising in heat transfer", Phys. Lett., A.360, pp. 109-113, 2006.

[10] L. Tao, H. Song and S. Chakrabarti, "Nonlinear progressive waves in water of finite depth-an analytic approximation", Clastal. Eng. 54, pp. 825-834, 2007.

[11] Z. Wang, L. Zou and H. Zhang, "Applying homotopy analysis method for solving differential- difference equation", Phys. Lett., A.369, pp.77-84, 2007.

[12] M. Hengjun, "A qualitative analysis of system with the Holling III functional response of predator to prey", Journal of Biomathematics, vol. 14, no.1, pp.12-19, 1999.

Received: June 06, 2013

Revised: September 15, 2013

Accepted: November 05, 2013

(C) Chen and $\mathrm{Yu}$; Licensee Bentham Open.

This is an open access article licensed under the terms of the Creative Commons Attribution Non-Commercial License (http://creativecommons.org/licenses/by-nc/3.0/) which permits unrestricted, non-commercial use, distribution and reproduction in any medium, provided the work is properly cited. 